\title{
Comparative rainfall data analysis from two vertically pointing radars, an optical disdrometer, and a rain gauge
}

\author{
E. I. Nikolopoulos ${ }^{1, *}$, A. Kruger ${ }^{1,2}$, W. F. Krajewski ${ }^{1}$, C. R. Williams ${ }^{2}$, and K. S. Gage ${ }^{2}$ \\ ${ }^{1}$ IIHR-Hydroscience \& Engineering, University of Iowa, Iowa City, Iowa, USA \\ ${ }^{2}$ Cooperative Institute for Research in Environmental Sciences, University of Colorado and Physical Sciences Division, \\ NOAA Earth System Research Laboratory, Boulder, Colorado, USA \\ * currently at: University of Connecticut, Storrs, USA
}

Received: 17 July 2007 - Revised: 31 October 2008 - Accepted: 6 November 2008 - Published: 16 December 2008

\begin{abstract}
The authors present results of a comparative analysis of rainfall data from several ground-based instruments. The instruments include two vertically pointing Doppler radars, S-band and X-band, an optical disdrometer, and a tipping-bucket rain gauge. All instruments were collocated at the Iowa City Municipal Airport in Iowa City, Iowa, for a period of several months. The authors used the rainfall data derived from the four instruments to first study the temporal variability and scaling characteristics of rainfall and subsequently assess the instrumental effects on these derived properties. The results revealed obvious correspondence between the ground and remote sensors, which indicates the significance of the instrumental effect on the derived properties.
\end{abstract}

\section{Introduction}

Theoretical and practical needs in hydrology and other geosciences stimulated much interest in research of rainfall fundamental properties. Problems of rainfall estimation using remote sensing and prediction at a wide range of temporal and spatial scales motivate efforts to improve our understanding of rainfall variability and dynamics. High resolution and quality observations are required for the task. Although most of our knowledge about rainfall comes from operational networks of rain gauges and weather radars, specialized instruments play increasing important role in filling resolution gaps (Krajewski and Smith, 2002; Krajewski et al., 2006).

In this paper we discuss results of rainfall characterization using four different collocated instruments that include a tipping-bucket rain gauge, a Parsivel optical disdrometer, and S-band and X-band vertically pointing Doppler radars (profilers). This physical setup of collocated instruments and

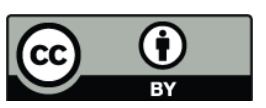

Correspondence to: A. Kruger

(anton-kruger@uiowa.edu) the fact that the profilers measure radar reflectivity essentially continuously in time and with high spatial resolution close to the ground represents the most favorable scenario for the intercomparison of the instruments. Thus, our emphasis is on addressing the issue of instrumental effects on the analyses rather than the methods themselves. Our characterization of variability includes autocorrelation, spectral, and moment scaling analyses in time from the recorded time series of data. Comparison of the results between the instruments allows exploring the instrumental-dependence of the derived rainfall properties.

We organized this paper as follows. In Sect. 2 we describe the experimental setup and some technical characteristics and the sampling strategies of the instruments we used in this study. In Sect. 3 we elaborate on the preparation of our data sets. Considerable data processing was required before we could begin our comparative analysis. In Sect. 4 we present the results of the different analysis we performed and in Sect. 5 we summarize and discuss the basic implications of our results.

\section{Experimental setup and instrumentation}

We operated two in-situ devices, i.e. a double tipping-bucket rain gauge and a Parsivel optical disdrometer and two remote sensors, i.e. an S-band and an X-band vertically pointing radar. The two VPRs and the Parsivel disdrometer were collocated within a $20 \mathrm{~m}$ radius (Fig. 1). The dual tippingbucket rain gauge platform was located some $200 \mathrm{~m}$ away from the rest of the equipment.

Figure 1 represents the differences in the spatial sampling scales between the sensors. The red dots represent the rain gauges, which have sampling area of about $325 \mathrm{~cm}^{2}$. The Parsivel has the sampling area of about $50 \mathrm{~cm}^{2}$. The yellow circles represent the VPR beam area that increases with height, and the yellow grid corresponds to the resolution

Published by Copernicus Publications on behalf of the European Geosciences Union and the American Geophysical Union. 


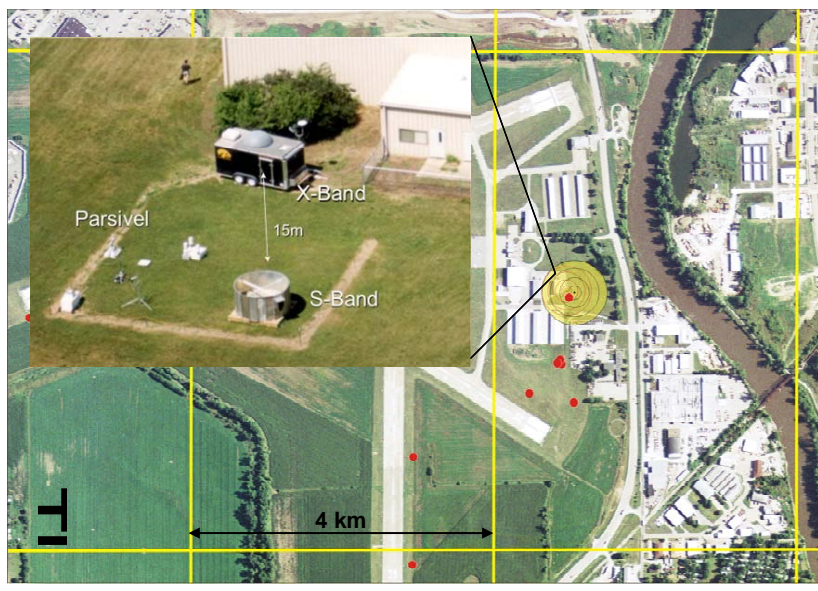

Fig. 1. Annotated aerial photograph of the Iowa City Municipal Airport. The yellow grid corresponds to the NEXRAD scanning radar grid, the yellow circles represent the VPR's beam area, and the red dots represent the rain gauges. The inset is an aerial photograph of the experimental site at the Iowa City Municipal Airport.

of Level II reflectivity data of the NEXRAD's WSR-88D weather radar located in Davenport, Iowa, i.e. some $80 \mathrm{~km}$ east of the Iowa City Municipal Airport. Note the differences in scales when comparing observations from different sensors.

Each of the instruments considered has unique advantages and limitations. Rain gauges are relatively inexpensive, reliable instruments, and the uncertainties associated with their observations are fairly well recognized (Habib et al., 1999, 2001; Ciach, 2003; Sieck et al., 2007). However, due to their point-like sampling area, the degree to which they represent larger spatial scales depends on the temporal scale of integration and remains an important subject of hydrologic studies (e.g. Bras and Rodriguez-Iturbe, 1985; Kitchen and Blackall, 1992; Krajewski et al., 2003; Ciach and Krajewski, 2006). Disdrometers share the same limitations as rain gauges but due to their ability to measure individual drops provide useful information about rain structure that cannot be obtained from rain gauges. The unit we used and its evaluation is discussed in detail by Krajewski et al. (2006).

Vertically pointing Doppler radars provide valuable information about precipitation structure and characterization (e.g. Atlas et al., 1973; Hauser and Amayenc, 1983; Williams et al., 1995). We operated an S-band (2.835 MHz) VPR (Fig. 1) that belongs to the NOAA Aeronomy Laboratory (Williams et al., 2005). The NOAA S-band samples the atmospheric profile with a spatial resolution of $60 \mathrm{~m}$ with the first range gate at $170 \mathrm{~m}$ above ground level. It has a parabolic dish diameter of $3.048 \mathrm{~m}$ and a beam width of $2.5^{\circ}$ that results in a sampling bin with a volume at the order of $340 \times 10^{3} \mathrm{~m}^{3}$ and a diameter of $44 \mathrm{~m}$ at $1 \mathrm{~km}$ height.

The second profiler that we operated is an X-band $(9.410 \mathrm{MHz})$ vertically pointing Doppler radar that it is
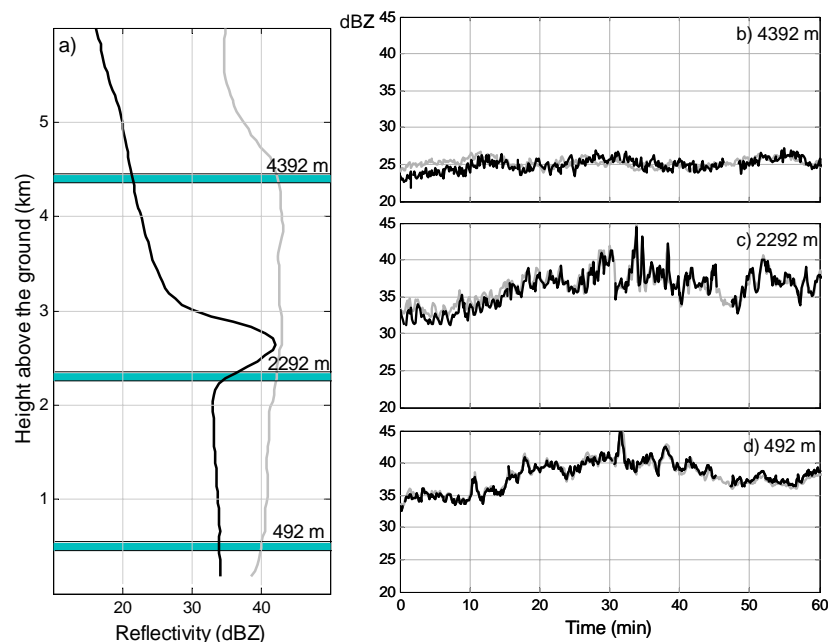

Fig. 2. Time series extracted from S-band profiler's data. Mean vertical profile of reflectivity (a), based on the storm events during 25 May (black) and 04 June (grey) 2002. The horizontal thick lines correspond to the three heights from which we extracted our time series. Time series of reflectivity (b), (c), and (d) correspond to heights 4392, 2292, and $492 \mathrm{~m}$ above the ground, respectively. The time series data are part of the storm event during 25 May 2002 and are shown for both S-band (black) and X-band (grey).

housed at the IIHR's Mobile Rainfall Observatory trailer (Fig. 1). The X-band's spatial resolution is $150 \mathrm{~m}$ and the lowest gate is located at $150 \mathrm{~m}$ above ground level. It has a parabolic dish diameter of $1.2 \mathrm{~m}$ protected and a beam width of $1.9^{\circ}$ that results in a sampling bin with a volume at the order of $439 \times 10^{3} \mathrm{~m}^{3}$ and a diameter of $33 \mathrm{~m}$ at $1 \mathrm{~km}$ height.

\section{Data preparation}

The dataset covers a period of 47 consecutive days beginning 11 May 2002 and ending 26 June 2002. While both VPRs operated most of the time, some gaps in the data record occurred because of computer and/or power failure. Comparison with the rain gauge data from the Iowa City Municipal Airport rain gauge network revealed that most of the missing data corresponded to no rain data. For our analysis, we either used the entire data set or focused on a number of selected storms (Table 1). For the analysis, we extracted time series (from the S-band profiler data) at three heights: close to the ground (492 m), close to but below the bright band (2292 m), and close to but above the bright band (4392 m) (Fig. 2).

Instrument calibration and quality control of the data are crucial issues, and we consider them individually for each instrument. Considerable data preparation was required to account for differences in spatial and temporal sampling scales, time shifts due to computer clock failure and/or drift, and other similar problems that may affect analyses results. The complete account of data preprocessing is described in 
Table 1. Selected storm events.

\begin{tabular}{|c|c|c|c|c|c|c|}
\hline \multirow{2}{*}{$\begin{array}{l}\text { Storm } \\
\text { Event }\end{array}$} & \multirow{2}{*}{$\begin{array}{l}\text { Start Date } \\
(\mathrm{mm} / \mathrm{dd} / \mathrm{yy})\end{array}$} & \multirow{2}{*}{$\begin{array}{c}\text { Start Time } \\
\text { UTC (hh:mm) }\end{array}$} & \multirow{2}{*}{$\begin{array}{l}\text { Duration } \\
\text { (min) }\end{array}$} & \multicolumn{2}{|c|}{ Rain Intensity from Gauge $(\mathrm{mm} / \mathrm{hr})$} & \multirow{2}{*}{$\begin{array}{c}\text { Rain Gauge } \\
\text { Accumulation } \\
(\mathrm{mm})\end{array}$} \\
\hline & & & & Mean & St. deviation & \\
\hline 1 & $05 / 11 / 02$ & $10: 20$ & 810 & 5.13 & 2.70 & 18.5 \\
\hline 2 & $05 / 25 / 02$ & $04: 57$ & 494 & 4.52 & 3.47 & 27.5 \\
\hline 3 & 06/02/02 & $10: 22$ & 397 & 3.12 & 4.03 & 14.6 \\
\hline 4 & 06/04/02 & $13: 30$ & 128 & 16.58 & 26.2 & 20.5 \\
\hline 5 & $06 / 11 / 02$ & 09:30 & 150 & 4.08 & 4.18 & 10.6 \\
\hline 6 & $06 / 13 / 02$ & $00: 30$ & 510 & 3.22 & 2.91 & 11.2 \\
\hline
\end{tabular}

Nikolopoulos (2004). In the following section we focus mainly on the profilers and we discuss some key issues that one should consider when comparing observations from different VPRs.

\subsection{Temporal and spatial scale matching}

The two radars have different sampling schemes and different temporal and spatial resolutions. Surprisingly, since the two instruments are collocated, these differences can have profound effect on a comparative analysis. For meaningful comparison, one has to average/combine and resample the data to get the time and spatial scales consistent.

As mentioned before, the S-band samples the vertical profile of the atmosphere with a spatial resolution of $60 \mathrm{~m}$, whereas the X-band has a spatial resolution of $150 \mathrm{~m}$. To make comparable spatial bins, we combined S-band radar bins to match the size of the X-band radar bins as closely as possible. More specifically, we combined two S-band bins to get a bin size of $120 \mathrm{~m}$ in order to compare with the $150 \mathrm{~m}$ bin size of the $\mathrm{X}$-band. When combining the two bins, $X$ and $Y$, one should remember that what the radar would measure if the actual bin size was $X+Y$, is not the simple arithmetic average of the measured variables from bin $X$ and bin $Y$. Let $Z_{i}\left(\mathrm{~mm}^{6} \mathrm{~m}^{-3}\right)$ be the reflectivity factor, measured by the radar in bin $i$. When we combine the two bins, $X$ and $Y$, the resulting bin $Q=X+Y$ will have

$Z_{Q}=\sum_{D=0}^{D_{\max }}\left(\frac{n_{X}(D)+n_{Y}(D)}{\text { volume of bin } Q}\right) D^{6} \Delta D$

where $n_{x}(D)$ and $n_{y}(D)$ is the number of drops (of diameter $D)$ in bin $X$ and $Y$ respectively. $D_{\max }$ is the maximum drop diameter and $\Delta D$ defines the differential drop diameter.

In our analysis we assumed that the two consecutive bins ( $X$ and $Y$ ) have equal volumes so we simplified Eq. (1) to

$$
\begin{aligned}
Z_{Q}= & \sum_{D=0}^{D_{\max }}\left(\frac{n_{X}(D)}{2 \times \text { volume of bin } X}+\frac{n_{Y}(D)}{2 \times \text { volume of bin } Y}\right) \\
& D^{6} \Delta D=\frac{Z_{X}+Z_{Y}}{2}
\end{aligned}
$$

Despite the matching of the sampling volumes in the vertical direction, there is still significant difference between the two volumes due to the difference in the beamwidth. The combined S-band bins compared with the X-band bin result in a volume difference of the order of $241 \times 10^{3} \mathrm{~m}^{3}$ at a height of $1 \mathrm{~km}$, which is equivalent to about $55 \%$ percent of the X-band bin volume at this height.

We try to mimic the S-band's sampling strategy by time averaging the X-band's samples. S-band samples continuously for about $8 \mathrm{~s}$, takes $1 \mathrm{~s}$ to write the data in the computer disc, and produces $9 \mathrm{~s}$ samples, whereas the $\mathrm{X}$-band produces samples every $0.25 \mathrm{~s}$. In order to eliminate this sampling scheme effect, we need to combine 32 of the X-band samples (32 samples $\times 0.25 \mathrm{~s} / \mathrm{sample}=8 \mathrm{~s})$ using the same averaging methodology that we described above to reproduce what the X-band would measure if it was sampling continuously for $8 \mathrm{~s}$. Basically, we are averaging in time by weighting again with the number of hydrometeors per sample. To account for the S-band's "dead time" of $1 \mathrm{~s}$, every time that we average $32 \mathrm{X}$-band samples we skip 4 samples (equal to $1 \mathrm{~s}$ ), and then we repeat the procedure.

\subsection{Radar calibration}

Both radars were calibrated to account for and minimize the systematic errors in our profiler observations. Clark et al. (2003) and Williams et al. (2003) used observations from a Joss-Waldvogel disdrometer to calibrate vertically pointing radar. We followed a similar technique but used observations from the collocated Parsivel disdrometer. The great advantage of using a disdrometer is that in a sense it extends the measurement of reflectivity factor to the surface (Clark et al., 2003), which allows the comparison with the profiler to be made directly on reflectivity space.

\subsection{Optimal comparison time scale}

When comparing observations between the profiler and the disdrometer, one should consider two major issues. The first involves the difference in the sampling volume between the two instruments. Since the reflectivity measured by the 


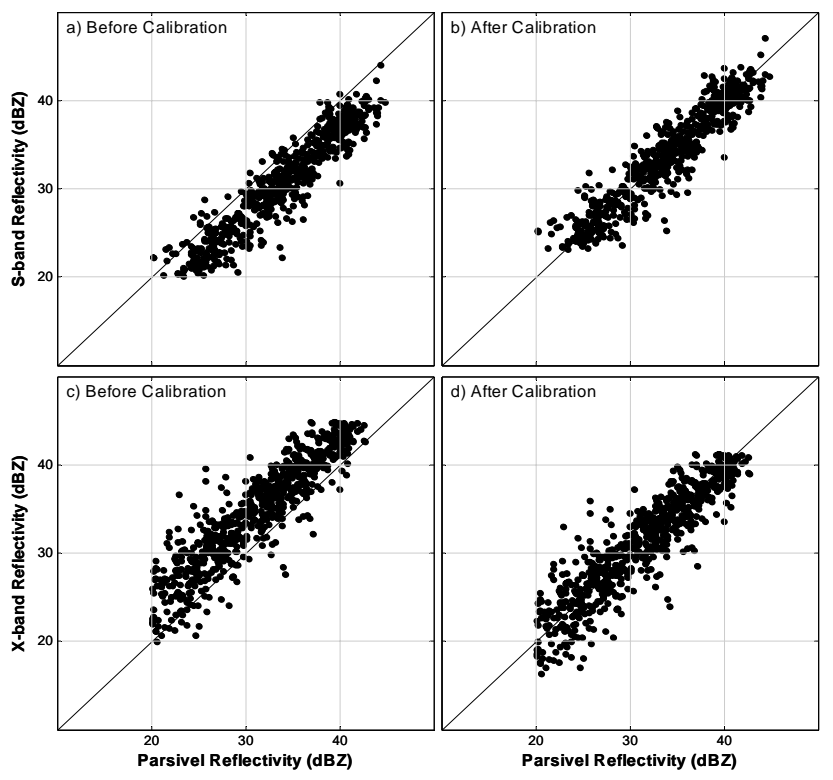

Fig. 3. Comparison between profiler (S-band and X-band) and Parsivel disdrometer reflectivity (dBZ) observations before calibration $(\mathbf{a}, \mathbf{c})$ and after calibration (b, d).

profiler is averaged over a significantly larger volume than that of the disdrometer (some nine orders of magnitude), it requires the disdrometer observations to be averaged over a time scale $(\Delta T)$ to best represent the volume-averaged reflectivity by the profiler. The second issue concerns the fact that the volume observed by the profiler at a certain height will take some time to reach the disdrometer at the ground. This time offset $(\tau)$ is related to the height of the observation and the falling speed of the drops inside the volume. Habib and Krajewski (2002) investigated these issues, but they compared radar-rainfall products with a resolution of $2 \times 2 \mathrm{~km}$ with rain gauge observations. We largely followed their methodology by using correlation-based analysis to determine the optimal comparison time scale.

After the time scale matching between the profilers, we had reflectivity samples every $9 \mathrm{~s}$ while the disdrometer produces $30 \mathrm{~s}$ samples. We chose the closest to the ground useable range-gate from the profiler to compare with the disdrometer. For the S-band, the first available gate is at $185 \mathrm{~m}$, and for the X-band, it is at $492 \mathrm{~m}$. The lower gates for the $\mathrm{X}$-band were contaminated with high signal noise, thus were not available for this analysis. We compared the observations from the profilers at these heights with the disdrometer observations shifted by $\tau$ and averaged over $\Delta T$ with a range for $\tau$ between -30 and $30 \mathrm{~min}$ and for $\Delta T$ between 1.5 and $30 \mathrm{~min}$. We optimized the correlation between the compared reflectivity values to determine the optimal $\tau$ and $\Delta T$.

Optimal time shifts were found around 1.5-3 min, which can be explained if you consider that an average drop $(1 \mathrm{~mm}$ diameter) with a fall speed of approximately $4 \mathrm{~m} \mathrm{~s}^{-1}$ takes about $2 \mathrm{~min}$ to reach the ground from a height of $492 \mathrm{~m}$. The highest correlation was obtained for $\Delta T$ around $1.5-5 \mathrm{~min}$. Habib and Krajewski (2002) showed an optimal range for $\tau$ values between $2-5 \mathrm{~min}$ and for $\Delta T$ values between 5 and $15 \mathrm{~min}$. The differences in our results are due to the fact that their radar observations were related to higher altitudes (requiring more time to reach the ground) and larger volumes (requiring larger integration scales) than our observations.

\subsubsection{Calibration scheme and results}

We calibrated the two radars after determining the optimal comparison time scale between the disdrometer and the profilers. As above, we extracted the data from the gates closest to the ground and compared them with the disdrometer observations. We compared the reflectivity values in $\mathrm{dBZ}$, and the calibration model we applied had the following form (Clark et al., 2003)

$Z_{\mathrm{CAL}}=Z_{\mathrm{RAW}}+C$

where $Z_{\mathrm{CAL}}(\mathrm{dBZ})$ is the profiler calibrated reflectivity, $Z_{\mathrm{RAW}}(\mathrm{dBZ})$ is the reflectivity measured by the profiler, and $C(\mathrm{~dB})$ is the calibration constant. When we calibrate, we simply want to adjust our values so that the mean difference between the reflectivity values from the disdrometer and the profiler goes to zero. Based on this, we obtained the calibration constant using the following formula

$C=E\left\{Z_{D}-Z_{\mathrm{RAW}}\right\}$

where $E$ is the expectation operator and $Z_{D}(\mathrm{dBZ})$ is the reflectivity value we obtained from the disdrometer data when we shifted them by $\tau$ and averaged them over $\Delta T$.

For the calibration procedure, we used data values above $20 \mathrm{dBZ}$ to avoid noisy data that were not removed by the filtering techniques and set an upper threshold at $45 \mathrm{dBZ}$ because of the limited sample size of values above this limit. We divided the data in $5 \mathrm{~dB}$ bins and classified all the values that were higher than three standard deviations within the bin as outliers and removed them from the data.

The results from the calibration procedure revealed that the S-band observations were underestimated (Fig. 3a) with a mean difference between the reflectivity values from the disdrometer equal to $3 \mathrm{~dB}$ and a standard deviation of $2 \mathrm{~dB}$ while the X-band's were overestimated (Fig. 3c) with a mean equal to $3.6 \mathrm{~dB}$ and a standard deviation $2.5 \mathrm{~dB}$. The distributions of differences between the profilers (S-band and X-band) and Parsivel (not shown here) have a similar normal-like shape, with that of the S-band being a little bit narrower due to the $0.5 \mathrm{~dB}$ difference of the standard deviations.

\section{Analysis of rainfall characteristics}

In our analysis, we compared rain rate time series from the four instruments. To obtain rain rate from the VPR measurements, we extracted the reflectivity time series from the 
height of $492 \mathrm{~m}$ and converted them to rain rate using the well known Z-R relationship of Marshall and Palmer (1948). In some cases we examined the 47 days dataset as one time series and in other cases we performed a storm-based analysis. We attempted to classify the storm events that passed overhead using the high-resolution data collected from the S-band VPR. This gave our analysis a further advantage because we were able to link certain rainfall characteristics with the type of rainfall. We generally followed the classification algorithm described by Williams et al. (1995), but whereas they used a $915 \mathrm{MHz}$ profiler for classification of precipitating clouds in the tropics, we used a $2835 \mathrm{MHz}$ profiler at a mid-western precipitation regime. Thus, in order to evaluate the effectiveness of the algorithm, we recalibrated the parameters and we used manually classified cases to compare against the algorithm's results.

\subsection{Autocorrelation analysis}

We performed a storm-based analysis to investigate the behavior of autocorrelation and the differences between the instruments for different rainfall events. We analyzed all the storm events described in Table 1. For the autocorrelation calculations, we used $4.5 \mathrm{~min}$ averaged rain rates from all instruments. We chose this as an optimal comparison time scale to minimize the effect of local random errors in rain gauge data and to keep the temporal resolution as high as possible. In the calculations we made an assumption of statistical stationarity of the rainfall process within each storm. While the validity of this assumption could be disputed, our focus is on the inter comparison of the instruments. Stationarity assumption is unlikely to affect our conclusions regarding ability of different instruments to characterize rainfall.

In Fig. 4, we present the results from the autocorrelation calculations from the four instruments and for the six storm events. Generally, there is very good agreement among the four instruments. The most prominent feature is the difference in autocorrelation for different storms. The time required for the autocorrelation to reach a minimum significant correlation value (1/e) varies from $5 \mathrm{~min}$ (Fig. 4d) to 40 min (Fig. 4b). Based on our precipitation classification results, the storms with fast decay of autocorrelation are associated with convective precipitation. This was expected, as the convective precipitation regimes are characterized by high temporal variability. On the contrary, stratiform type of precipitation is more homogeneous and is associated with slow decay of autocorrelation. The differences between the instruments do not appear to have a clear relationship with the precipitation type. Another noticeable feature is that, in most cases, the autocorrelation from the VPR is higher than the one from the rain gauge and disdrometer. This effect is related to the differences in sampling volume. The profiler's observations are averaged over a much larger volume, which results in an increase in the correlation. Instead of a smooth continuous decrease of the correlation with time, in
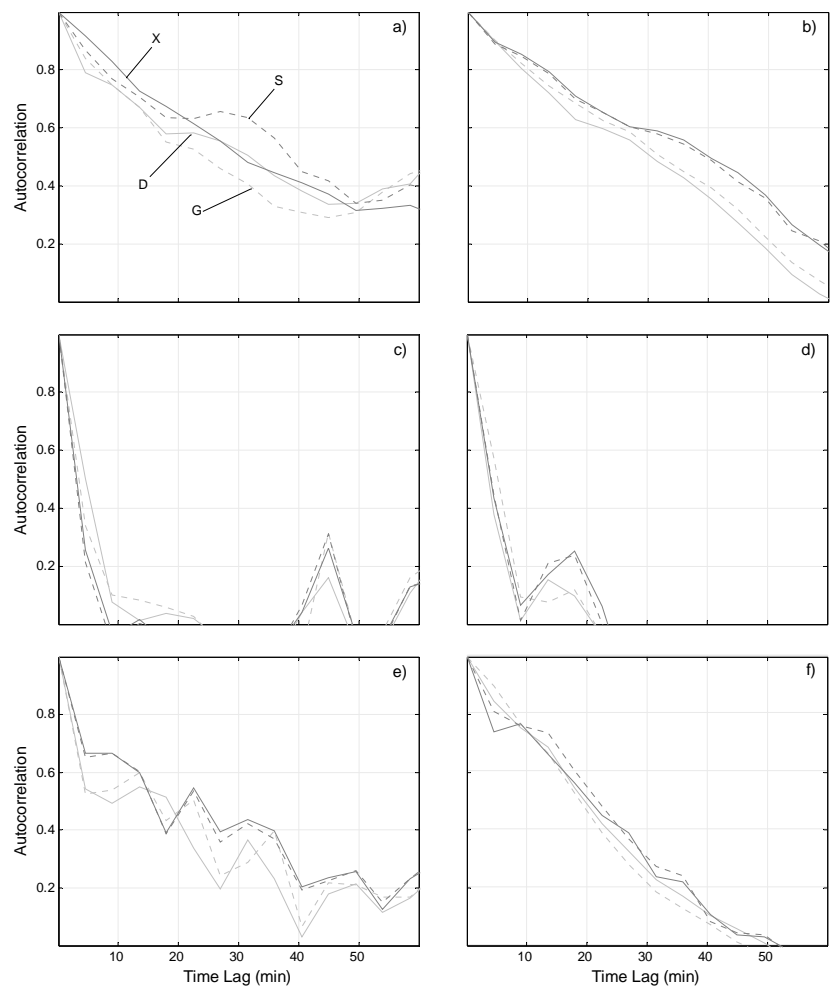

Fig. 4. Autocorrelation based on $4.5 \mathrm{~min}$ averaged rain rate observations from rain gauge $(G)$, Parsivel disdrometer (D), S-band VPR (S), and X-band VPR (X). The six storm events we analyzed include 11 May (a), 25 May (b), 2 June (c), 4 June (d), 11 June (e), and 13 June (f), 2002.

some cases we observe consecutive increases and decreases of the autocorrelation (Fig. 4e), which are mainly associated with oscillations in the rainfall time series.

We continued our analysis by comparing the highresolution data from the S-band and X-band VPR. We focused mainly on two different storm events, which we classified as stratiform (25 May 2002) and convective (4 June 2002) based on the results of our classification algorithm. In Fig. 5, we present the results from autocorrelation calculations from both radars, at the three different heights and for both stratiform and convective cases. Both radars are in very good agreement, with that of the S-band being consistently higher than the X-band. Though the difference is insignificant it might be caused by difference in sampling volumes. The S-band sampling volume is 55\% larger than that of $\mathrm{X}$ band, which might explain the slightly higher correlation values. The autocorrelation decreases with increasing height and is more evident in the stratiform case. One could argue that the use of a Marshall-Palmer Z-R above the melting layer (i.e. snow observations) might be inappropriate. However, a simple numerical exercise which included the comparison between autocorrelation values based on snow derived Z-R's (Muramoto et al., 2003) and the Marshall-Palmer 

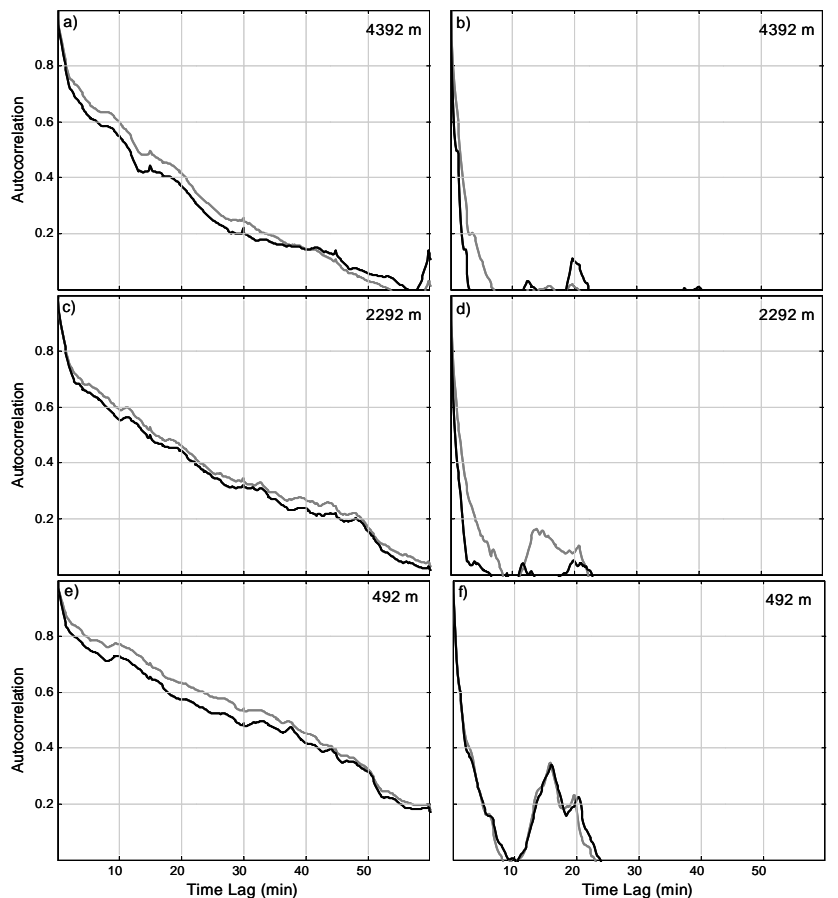

Fig. 5. Autocorrelation of rain rate time series derived from $\mathrm{S}$ band (grey) and X-band (black) $9 \mathrm{~s}$ resolution data for three different heights and for two different events. The stratiform event (a), (c), (e) during 25 May 2002 and the convective event (b), (d), (f) during 4 June 2002.

Z-R revealed no significant difference that would alter our conclusions.

Note that Figs. $4 \mathrm{~b}$ and $5 \mathrm{e}$ correspond to the same event but there is a small difference in autocorrelation from the two profilers. The reason is that in the case of Figure $4 \mathrm{~b}$ we used rain rates averaged over $4.5 \mathrm{~min}$, which resulted in higher correlation values compared to that of Fig. 5e which was based on $9 \mathrm{~s}$ rain rates.

\subsection{Spectral analysis}

We also performed spectral analysis on our precipitation signal to investigate the variability of precipitation as a function of scale. Following a methodology similar to Fabry (1996), we calculated the power spectrum of our rain rate time series retrieved from both profilers. We performed the power spectrum calculations on a daily basis for the two selected events. A daily precipitation signal consists of 9600 sample points ( 1 sample per $9 \mathrm{~s})$. To compute the power spectra of this signal we used a length of Fast Fourier Transformation points equal to 1024 and applied a Hanning window with a 50\% overlap. In Fig. 6, we present the results obtained for the different heights and different storm events.

The power spectra of a signal are related to its scaling properties. For multiscaling or multiaffine fields, the Fourier

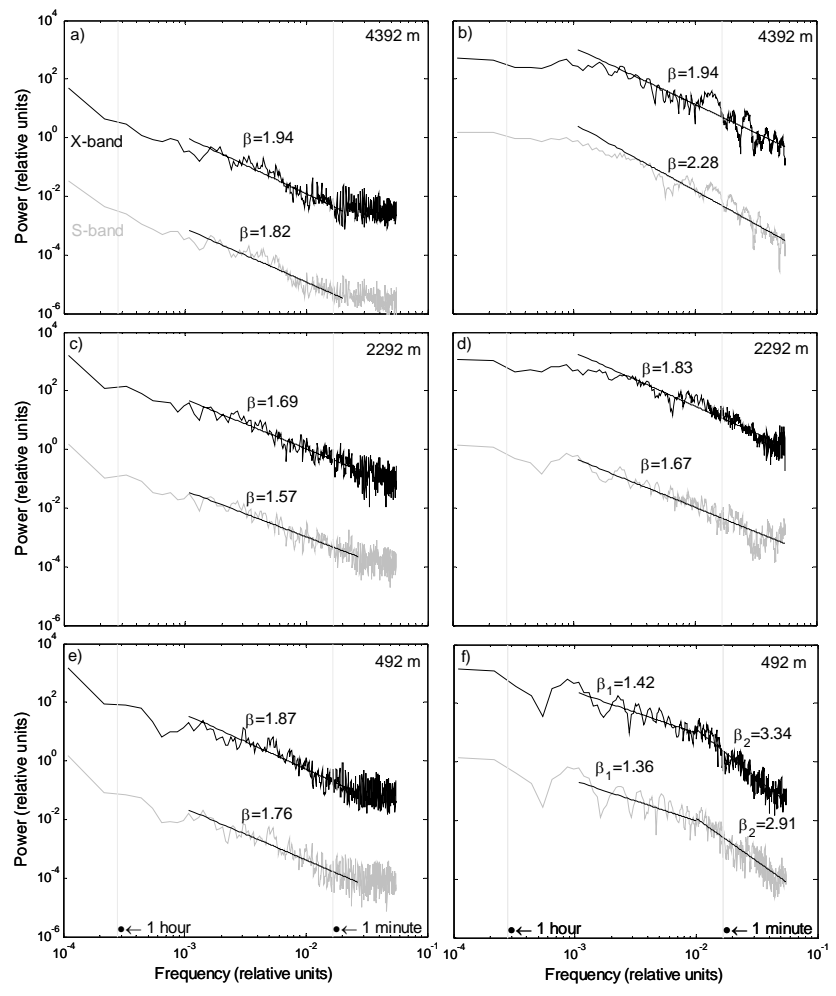

Fig. 6. Power spectra of precipitation rates as derived by the S-band (grey) and X-band (black) VPR at three different heights for two different storms. The stratiform event (a), (c), (e), during 25 May 2002 and the convective (b), (d), (f) during 4 June 2002. Note that we shifted (multiplied by a factor of $10^{2}$ ) the X-band spectra for better display.

power spectrum exhibits a power law behavior of the form

$P \sim f^{-\beta}$

where $P$ is the power in relative units, $f$ is the frequency in relative units, and $\beta$ is the power law exponent usually determined by the slope of the spectrum on a log-log plot (Harris et al., 1998). One basic assumption that we imply in order to perform the spectral analysis is that our signal is stationary. Otherwise, the Fourier power spectrum is not defined. The spectra we obtained from both radars are in good agreement for all the heights and both storm events. We estimated the exponent $\beta$ based on least squares linear regression, and the results revealed that the $\mathrm{X}$-band data were consistently associated with higher values, except in the case of $4392 \mathrm{~m}$ height for the convective storm.

The range of the data that we used to fit the lines varied from case to case depending on whether one or more slopes could best describe the power spectra. More specifically, for the stratiform case at 492 and $2292 \mathrm{~m}$, we fitted the straight lines for periods longer than $30 \mathrm{~s}$ and for periods longer than $1 \mathrm{~min}$ at the $4392 \mathrm{~m}$. For the convective case, we fitted two lines for periods longer and shorter than $1.5 \mathrm{~min}$ 
for the $492 \mathrm{~m}$ and used the whole spectra to fit the lines for the heights of 2292 and $4392 \mathrm{~m}$.

The stratiform event we examined revealed a break in the power law behavior at scales around $30 \mathrm{~s}$ that was followed by a flattening of the power spectra. This indicates a near white-noise variability at smaller time scales. Similar behavior of the spectra is not apparent in Georgakakos et al. (1994), but Fabry (1996) obtained similar results with the main difference being that he observed the break in the slope at smaller time scales (around $5 \mathrm{~s}$ ). This flat regime of the spectra is translated as a lack of precipitation structure at these small time scales. Fabry (1996) suggested that the spreading of hydrometeors when submitted to wind shear, because of their differential fall speeds, will result in an increasing loss of precipitation structure with distance below the generating point of hydrometeors, which can explain the nearly white-noise variability at small scales. The break between the power law and the almost flat spectrum occurs at larger scales above the melting layer (Fig. 6a) than below it (Fig. 6c), which indicates, as Fabry (1996) suggested, that the melting layer reintroduces small-scale structure into the precipitation field.

In the convective case, we did not observe a similar regime of near flat spectra, which indicates that convective precipitation exhibits structure at smaller scales than stratiform. In addition, the fact that precipitation structure varies with precipitation type reinforces the likelihood that the observed flat spectra regime for the stratiform case is not an artifact of the instrument. The most prominent feature in the convective case is that the spectra from precipitation signal close to the ground (Fig. 6f) reveal a break in the slope at a time scale close to $1.5 \mathrm{~min}$. The spectral regime at time scales smaller than the break point is characterized by a much steeper slope (approximately equal to 3) than that for larger time scales (approximately equal to 1.5). These two different regimes suggest that two different scaling regimes govern our rainfall signal. The reason that this break does not appear in the higher altitudes is unclear.

The results from our spectral exponent estimation for time scales larger than $1 \mathrm{~min}$, for the heights of 492 and $2292 \mathrm{~m}$ and for both events, vary between 1.36 and 1.87 and are in general agreement with the one obtained by Georgakakos et al. (1994). These exponents are close to the classical Kolmogorov $f^{-1.66}$ power law relationship, which represents the spectrum predicted for the fluctuations of a passive scalar introduced into a turbulent fluid (Olsson et al., 1993). The latter suggests that precipitation structure at these scales is turbulence driven.

\subsection{Moment scaling analysis}

Scaling properties of rainfall in spatial and temporal domain is a topic of growing interest in the last decades. Researchers have used a variety of approaches to investigate the scaling properties of the rainfall process, with multifractal analysis being the most popular (Olsson et al., 1993; Harris et al., 1997, 1998; de Lima and Grasman, 1999; Kiely and Ivanova, 1999). Following the same spirit with previously we try to investigate the instrumental effect on the derived scaling properties of rainfall by comparing the results we obtained from the four instruments.

The concept of temporal scale invariance (scaling) holds for the rainfall field when the moment $M_{q}$ of order $q \geq 0$ of the field at a temporal scale, $t$, is related to the same moment $M_{q}$ at a different scale $\lambda t$ according to the relationship

$M_{q}(\lambda t)=\lambda^{K(q)} M_{q}(t)$

where $K(q)$ is the scaling exponent or the so-called moment scaling function and $\lambda$ is the scaling factor that we define as the ratio of the given scale $t_{j}$ and a reference scale $t_{0}$,

$\lambda=\frac{t_{j}}{t_{0}}$

Based on the above, $\lambda$ is always $\geq 1$. The moment of order $\mathrm{q}$ of a discrete random variable $X$ is defined as

$$
M_{q}=\sum_{\text {all } x_{i}}\left(x_{i}\right)^{q} p_{X}\left(x_{i}\right)
$$

where $x_{i}$ is the value of $X$ at point $i$ and $p_{X}$ is the probability mass function of $X$ (Kottegoda and Rosso, 1997). Based on Eq. (8), we calculated the moments of order $q(0 \leq q \leq 5)$ starting at the finest resolution of our data and then degraded the resolution by averaging and recalculated the moments. By repeating this process for different resolutions, we calculated $M_{q}$ for a range of different scales. In order to obtain the value of $K(q)$, we plotted the values of $\log M_{q}$ against the $\log$ of scaling factor, $\log \lambda$, and fitted a line based on least squares regression to determine the slope of the line. The value of the slope is the $K(q)$ value.

As the first step of our moment scaling analysis, we used all 47 days of collected data. In order to determine the range of scales over which the scale invariance holds, we calculated $M_{q}$ from our data from time scales starting at $4.5 \mathrm{~min}$ up to approximately 12 days. Because the finest temporal resolution of the data differs with instrument, we used $4.5 \mathrm{~min}$ averaged rain rates to compare the four instruments on the same time scales. In Fig. 7, we present the results from all instruments. As we can observe, there is a clear break in the scaling, common for all the calculated moments, at time scales around $2.4 \mathrm{~h}$, which suggests that there are two different scaling regimes within the range of scales we investigated that govern the rainfall dynamics. This feature is more distinct for the results from the VPRs. Olsson et al. (1993) observed similar breaks in the scaling of rainfall and suggested that these breaks correspond to characteristic time periods when changes in the temporal structure of the rainfall distribution occur. The average duration of wet periods in our data was $1.6 \mathrm{~h}$ with $1.5 \mathrm{~h}$ standard deviation.

For the calculation of the $K(q)$, we estimated the slope of the fitted lines for scales up to the breaking point $(2.4 \mathrm{~h})$. 

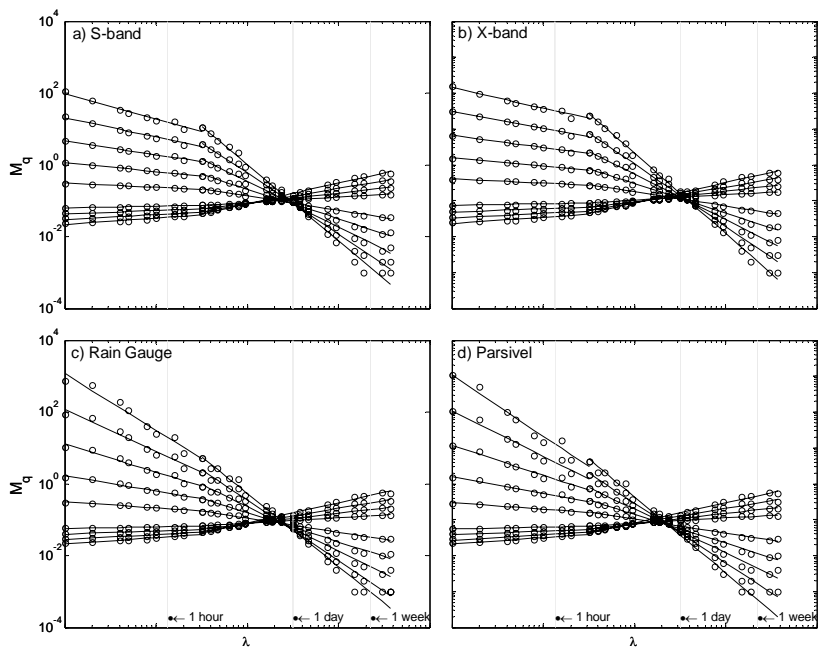

Fig. 7. Log-log plot of moments $M_{q}$ of order $q$ versus the scaling factor $\lambda$. Moments were calculated for time scales starting at $4.5 \mathrm{~min}$ up to 47 days. Note the break in the slope around $2.4 \mathrm{~h}$ that suggests the existence of two scaling regimes. The moments displayed (bottom to top) are of order $q$ equal to $0.2,0.4,0.6,0.8,1.5$, $2,2.5,3$ and 3.5 respectively.

In Fig. 8, we present the results we obtained from the four instruments. For $q<1.5$, all the instruments are in excellent agreement, but for $q>1.5$ there is a clear difference between the resulting $K(q)$ curves from the ground sensors (rain gauge and disdrometer) and the remote sensors (S-band and X-band VPR), with the first having a much steeper curve compared to the two profilers. A steep $K(q)$ curve means that the moments of the underlying process differ greatly with different scales. Thus, the results we obtained from the four instruments suggest that the rain rates derived from the ground sensors show higher variability. This is not surprising if we consider the fact that the rain rates derived from the profiler's measurements are obtained by averaging over a sampling volume much larger than the one of the ground sensors, which results in a smoother rain rate signal.

The $K(q)$ curves for both profilers show highly linear behavior whereas both $K(q)$ that correspond to the ground sensors exhibit nonlinear behavior (at least at some extent). The reasons for the differences in the observed $K(q)$ are not clear, but the fact that there are certain similarities between the ground sensors and the remote sensors suggests that the differences in sampling principles that characterize the two groups of sensors might be a possible cause. One can speculate that the constant Marshall-Palmer Z-R relationship that we used to derive the rain rates from the VPR's observations can have a significant effect in the scaling properties. In order to investigate this, we repeated the scaling calculations for the S-band data but this time instead of using a single Z$\mathrm{R}$ we used a storm-based Z-R derived from the disdrometer data. The results, shown in Fig. 8, suggest that although there

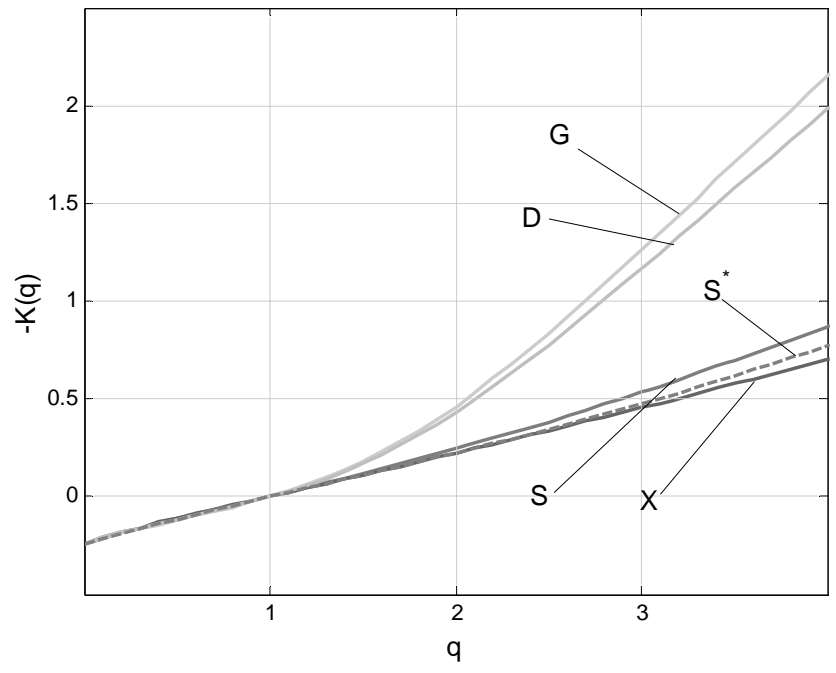

Fig. 8. Scaling function $K(q)$ versus moment of order $q$ for S-band (S), X-band (X), Parsivel (D) and rain gauge (G). The calculation of $K(q)$ was based on the 47 days of data and for time scales starting from $4.5 \mathrm{~min}$ until approximately $2.4 \mathrm{~h}$. The $\mathrm{S}^{*}$ and $\mathrm{S}$ curves corresponds to rainrates derived from S-band data based on different storm-based Z-R relationships (derived from disdrometer) and a constant Marshall-Palmer Z-R, respectively.

is some effect of the different Z-Rs, it cannot explain the big difference between ground and remote sensors.

Since the above analysis was based on all 47 days of data, the scaling properties we derived result from a "mixing" of different storm events. In order to investigate the dependence of the derived scaling properties with the storm event as well as the differences between the instruments with storm event, we continued our analysis on a storm basis. We followed exactly the same methodology we described above for the same 6 storms we used for the autocorrelation analysis.

In Fig. 9, we present the observed $K(q)$ curves for each storm event and for all instruments. Again the $K(q)$ values were calculated for scales up to $2.4 \mathrm{~h}$. The storm events of 2 June, 4 June, and 11 June 2002 are associated with much steeper curves that indicate higher variability. All instruments are in very good agreement up to the second order moment, but for higher moments there is an apparent difference between instruments. These differences are not consistent with a storm event, which suggests their dependence on precipitation type. However, we should keep in mind that the higher order moments $(q>2)$ are strongly affected by the higher values of rain rate, which means that the observed difference between the $K(q)$ curves for high order moments might in fact reflect the differences in these few extreme rain rate values recorded from the instruments. Especially for the rain rates derived from the VPR measurements, the chosen $\mathrm{Z}$ $\mathrm{R}$ relationship strongly affects the magnitude of the extreme rain rate values. Finally, as we have mentioned before, due to the larger sampling volume, the profilers record a smoother 

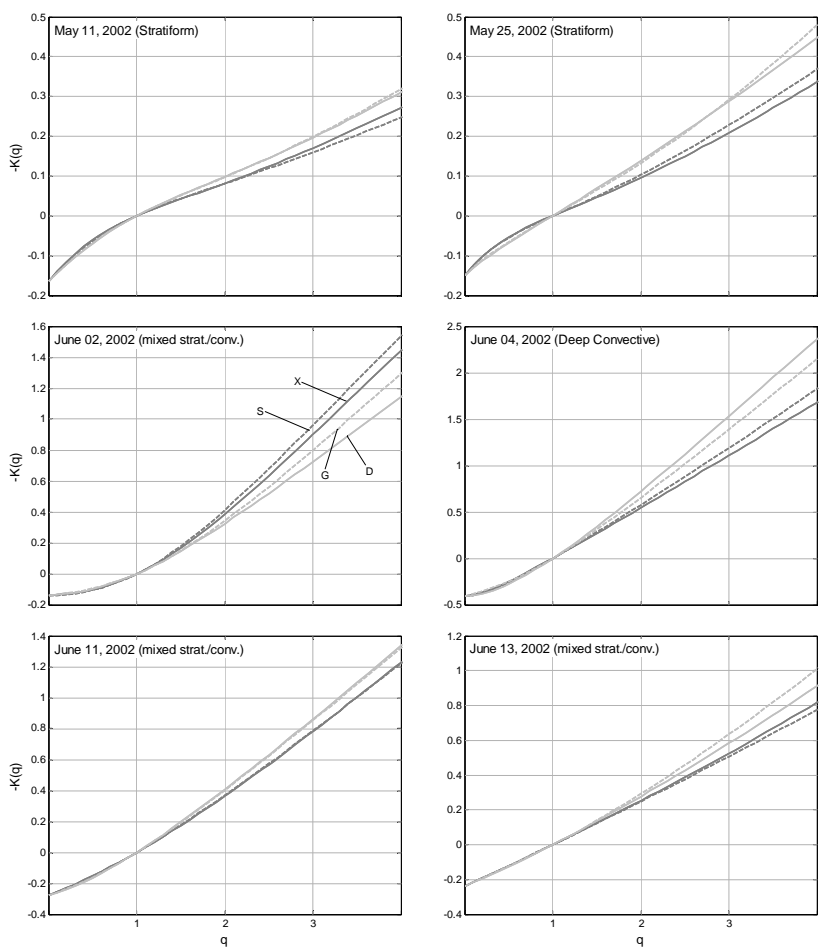

Fig. 9. Observed $K(q)$ curves for the six storm events and for all instruments. Note that the $y$-axis scale is different in order to display the details of the curve's shape.

rainfall signal with lower extreme values than those recorded from the ground sensors. This explanation is reinforced by the fact that, in most cases, ground and remote sensors exhibit similar behavior.

The shape and magnitude of the observed $K(q)$ curves differ significantly with storm event (for all instruments), which suggests that scaling properties of rainfall depend strongly on rainfall type. Thus, it is somewhat inappropriate to try to derive the scaling characteristics of rainfall from the "mixing" of these storms. We did this in our initial approach by using all 47 days of data, and although someone can use the results as an approximation of the scaling characteristics of rainfall, in our storm-based analysis we demonstrated that this approximation can sometimes differ significantly.

As the final step of our scaling analysis, we investigated the scaling properties of rainfall at different altitudes for stratiform and convective precipitation. We used the rain rates derived from the S-band and X-band VPR at 492, 2292, and $4392 \mathrm{~m}$ during the storm events of 25 May and 4 June 2002 that were mainly classified as stratiform and convective, respectively. In Fig. 10, we present the calculated $K(q)$ curves for both storms and for all altitudes.

For the stratiform event, the $K(q)$ curves for S-band and $\mathrm{X}$-band are almost identical at each height but they differ with height. More specifically, the $K(q)$ curves appear to be steeper at the altitude of $2292 \mathrm{~m}$ (below the melting layer)
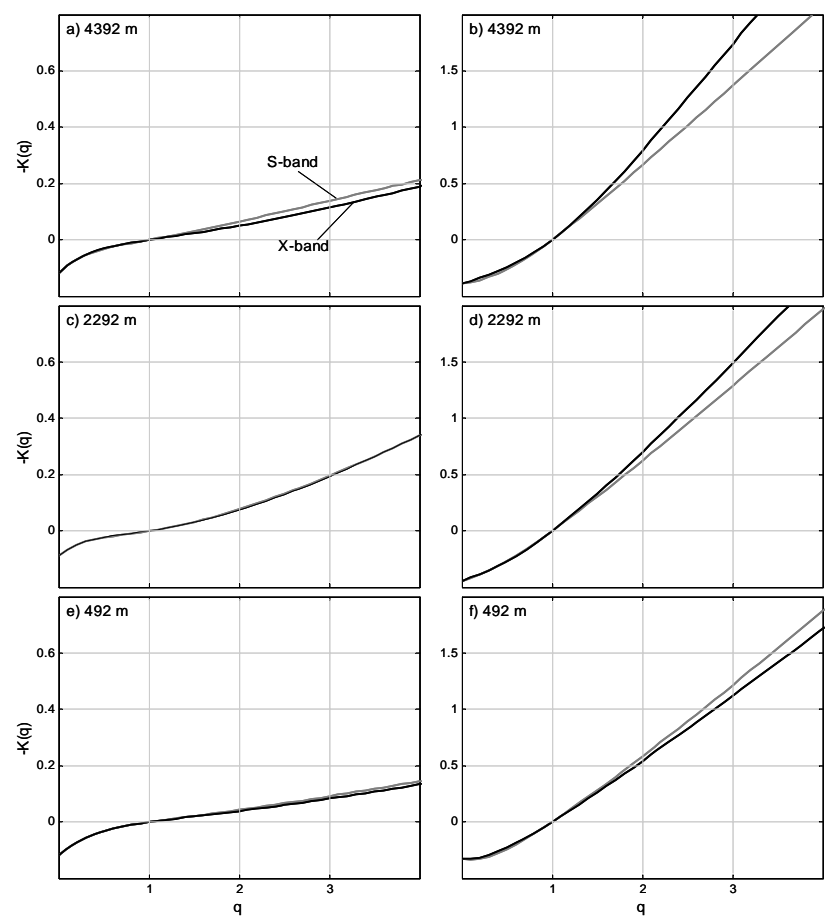

Fig. 10. Calculated $K(q)$ curves based on rain rates derived from Sband and X-band data at three different altitudes, for the stratiform (25 May) and convective (4 June) storm event. Note that the y-axis scale is different between the stratiform (a), (c), (e) and convective (b), (d), (f) event.

than at $492 \mathrm{~m}$ (close to the ground), which suggests increasing variability with altitude, but at $4392 \mathrm{~m}$ (above the melting layer) the variability decreases and results in a less steep $K(q)$ curve.

The convective event is associated with much steeper $K(q)$ curves, which is not surprising since it corresponds to what we currently know about the higher variability of convective precipitation. Moreover, we can observe that the $K(q)$ values increase with altitude as well as with the difference between the two radars. One could speculate that the attenuation effect which increases with altitude and is much more severe for the X-band (shorter wavelength) could explain these differences in some degree. However in our case we investigated the significance of the impact of attenuation effect on our observations (by calculating the difference in measured reflectivity between S-band and X-band for different altitudes) and the results (not shown here) suggest that there is no significant impact on X-band's observations due to atmospheric attenuation. The difference between the two profilers can be possibly attributed (to a certain degree) to the difference in sampling volumes, which increases with altitude. 
Our scaling analysis results might be subject to significant uncertainty due to the small size of the sample of storms that we used. The impact of this issue is not well recognized in the literature and the potential problem is difficult to overcome as deployments of several collocated instruments are limited in time by duration of field experiments and funding. Still, since our emphasis is on the inter comparison of instrumental ability to characterize rainfall, potential errors in some specific characteristics are of lesser importance.

\section{Closing remarks and discussion}

Technological advances in the field of rainfall studies over the last few decades have led to the development of such innovative instruments as disdrometers, scanning radars, and profilers. Researchers utilize these new instruments to more effectively study several aspects of rainfall (e.g. estimation, statistical properties). However, all instruments are subject to a number of different sources of errors that introduce uncertainty in the estimates of rainfall and the properties derived from their observations. In this study, we investigated certain properties of rainfall and tried to explain central issues relative to the instrumental-dependence of our results. Below, we summarize our main conclusions and discuss issues that require further investigation.

A number of factors (calibration, difference in temporal and spatial sampling etc.) must be considered in order to make a comparative analysis among different instruments meaningful. We addressed these issues and we described a methodology to account for most of them, but we acknowledge that there are still limitations regarding our approach (e.g. imperfect calibration and spatial matching).

Despite these differences between instruments, the results from the autocorrelation analysis showed very good agreement. This suggests that all instruments were able to capture and represent the temporal variability of rainfall. The profilers allowed us to investigate this variability at a vertical extent and obtain useful information for the stratiform and convective types of precipitation. However, our spectral analysis results were based only on 2 storm events, and further analysis of more cases is therefore needed to verify a specific pattern of the temporal structure of rainfall in the stratiform and convective regimes.

The scaling investigation revealed certain similarities in the results from ground and remote sensors. Although we speculate that this is due to the differences in sampling volume, further analysis is needed. A possible approach to demonstrate the effect of sampling volume would be to average several sampling bins from the profiler data and repeat the scaling calculations. The storm-dependence of the differences that was revealed suggests that the instruments do not represent all storms equally well, and although one would expect that the stratiform would show the best results, that was not always the case. One can argue that the storm-based analysis is associated with the drawback of a small sample size and the effect that the few high values have to higher order moments can possibly explain the discrepancies observed but we observed distinct differences even for $q \leq 2$.

These differences also imply caution that one should use when attempting to translate results obtained in the time domain to the spatial domain. While vertically pointing radars provide more information about rainfall structure than ground-based in-situ devices such as rain gauges and disdrometers, the fundamental issues in translating the temporal into spatial domain remain. For a discussion of these issues an interested reader is referred to Fabry (1996), for example.

Acknowledgements. The authors acknowledge support from NSF Grant EAR-0409738. NOAA participation was supported in part by a grant from the NASA Precipitation Measurement Mission. W. Krajewski acknowledges partial support of the Rose \& Joseph Endowment.

Edited by: J. de Lima

Reviewed by: three anonymous referees

\section{References}

Atlas, D., Srivastava, R. C., and Sekhon, R. S: Doppler radar characteristics of precipitation at vertical incidence, Rev. Geophys. Space Phys., 11, 1-35, 1973.

Bras, R. L. and Rodriguez-Iturbe, I.: Random functions and hydrology, Reading, Massachusetts, Addison-Wesley Publishing Company, 1985.

Ciach, G. J.: Local random errors in tipping-bucket rain gauge measurements, Adv. Water Resour., 22, 585-595, 2003.

Ciach, G. J. and Krajewski, W. F.: Analysis and modeling of spatial correlation structure of small-scale rainfall in Central Oklahoma, Adv. Water Resour., 29, 1450-1463, 2006.

Clark, W. L., Gage, K. S., Carter, D. A., Williams, C. R., Johnston, P. E., and Tokay, A.: Calibration issues using impact disdrometers for calibration of Doppler radar profilers, paper presented at 12th Symp. on Meteor. Observ. and Instrum., Amer. Meteor. Soc., California, abstract number P1.16, 2003.

de Lima, M. I. P. and Grasman, J.: Multifractal analysis of 15-min and daily rainfall from a semi-arid region in Portugal, J. Hydrol., 220, 1-11, 1999.

Fabry, F.: On the determination of scale ranges for precipitation fields, J. Geophys. Res., 101, 12 819-12 826, 1996.

Georgakakos, K. P., Carsteanu, A. A., Sturdevant, P. L., and Cramer, J. A.: Observation and analysis of Midwestern rain rates, J. Appl. Meteorol., 33, 1433-1444, 1994.

Habib, E. and Krajewski, W. F.: Uncertainty analysis of the TRMM ground-validation radar-rainfall products: application to the TEFLUN-B field campaign, J. Appl. Meteorol., 41, 558-572, 2002.

Habib, E., Krajewski, W. F., Nespor, V., and Kruger, A.: Numerical simulation studies of rain gauge data correction due to wind effect, J. Geophys. Res., 104, 19723-19734, 1999.

Habib, E., Krajewski, W. F., and Kruger, A.: Sampling errors of tipping-bucket rain gauge measurements, J. Hydrol. Eng., 6, 159-166, 2001. 
Harris, D., Seed, A., Menabde, M., and Austin, G.: Factors affecting multiscaling analysis of rainfall time series, Nonlin. Processes Geophys., 4, 137-156, 1997, http://www.nonlin-processes-geophys.net/4/137/1997/.

Harris, D., Menabde, M., Seed, A., and Austin, G.: Breakdown coefficients and scaling properties of rain fields, Nonlin. Processes Geophys., 5, 93-104, 1998,

http://www.nonlin-processes-geophys.net/5/93/1998/.

Hauser, D. and Amayenc, P.: Exponential Size Distributions of Raindrops and Vertical Air Motions Deduced from Vertically Pointing Doppler Radar Data Using a New Method, J. Appl. Meteorol., 22, 407-418, 1983.

Kiely, G. and Ivanova, K.: Multifractal analysis of hourly precipitation, Phys. Chem. Earth (B), 24, 781-786, 1999.

Kitchen, M. and Blackall, R. M.: Representativeness errors in comparisons between radar and gauge measurements of rainfall, J. Hydrol., 134, 13-33, 1992.

Kottegoda, N. T. and Rosso, R.: Statistics, Probability, and Reliability for Civil and Environmental Engineers, The McGraw-Hill Companies, Inc., 1997.

Krajewski, W. F. and Smith, J. A.: Radar Hydrology: rainfall estimation, Adv. Water Resour., 25, 1387-1394, 2002.

Krajewski, W. F., Ciach, G. J., and Habib, E.: An analysis of smallscale rainfall variability in different climatological regimes, Hydrol. Sci. J., 48(2), 151-162, 2003.

Krajewski, W. F., Kruger, A., Caracciolo, C., Golé, P., Barthes, L., Creutin, J.-D., Delahaye, J.-Y., Nikolopoulos, E. I., Ogden, F., and Vinson, J.-P.: DEVEX-Disdrometer Evaluation Experiment: Basic results and implications for hydrologic studies, Adv. Water Resour., 29, 311-325, 2006.
Marshall, J. S. and Palmer, W.: The distribution of raindrops with size, J. Meteorol., 5, 165-166, 1948.

Muramoto K., Ebisu, S., Servomaa, H., Kubo, M., and Shiina, T.: ZR Relationship for Individual Snowfall and its Evaluation, SICE Annual Conference in Fukui, Japan, 2003.

Nikolopoulos, E. I.: Analysis of high resolution data of vertical structure of rainfall, M. S. thesis, Univ. of Iowa at Iowa City, Iowa, 2004.

Olsson, J., Niemczynowicz, J., and Berndtsson, R.: Fractal analysis of high-resolution rainfall time series, J. Geophys. Res., 98, 23 265-23 274, 1993.

Sieck, L. C., Burges, S. J., and Steiner, M.: Challenges in obtaining reliable measurements of point rainfall, Water Resour. Res., 43, W01420, doi:10.1029/2005WR004519, 2007.

Williams, C. R., Ecklund, W. L., and Gage, K. S.: Classification of precipitating clouds in the tropics using $915-\mathrm{MHz}$ wind profilers, J. Atmos. Ocean. Tech., 12, 996-1012, 1995.

Williams, C. R., Johnston, P. E., Clark, W. L., Gage, K. S., Carter, D. A., and Kucera, P. A. Calibration of scanning radars using vertically pointing profilers and surface disdrometers, paper presented at 12th Symp. on Meteor. Observ. and Instrum., Amer. Meteor. Soc., California, abstract number 11.3, 2003.

Williams, C. R., Gage, K. S., Clark, W., and Kucera, P.: Monitoring the reflectivity calibration of a scanning radar using a profiling radar and a disdrometer , J. Atmos. Ocean, Tech., 22, 1004-1018, 2005. 